\title{
On the nature of relationship between self-regulation and lexical competence
}

\author{
Sanja Marinov \\ Faculty of Economics, Business and Tourism, Split, Croatia \\ smarinov@efst.hr \\ Višnja Pavičić Takač \\ Faculty of Humanities and Social Sciences, Osijek, Croatia \\ vpavicic@knjiga.ffos.hr
}

\section{Introduction}

The complex construct of lexical competence has generated a plethora of approaches to modelling its multidimensionality. Thus, separate traits models itemize and describe all aspects and dimensions of lexical knowledge (cf. Nation, 2001; Milton 2009), global traits models attempt to encompass a few essential dimensions that represent the main characteristics of lexical knowledge and are unrelated to the features of individual lexemes (cf. Daller et al., 2007; Bulté et al. 2008). In addition, the models may include dimensions to account for the difference between the receptive and productive aspects of lexical competence or for word networks created in a person's mental lexicon. Productive vocabulary knowledge can be further divided in: controlled, which involves "producing words when prompted by a task" (e.g. cloze and translation tests) and free, dealing with the "use of words at one's free will, without any specific prompts for particular words", as is the case of free writing (Laufer, 1998, p. 257).

An inspection of these models reveals that the number of proposed dimensions differs, but most models recognize the theoretical constructs of size, width and depth of vocabulary knowledge which are statistically measured by the corresponding behavioural constructs of lexical diversity, lexical density or productivity, and lexical sophistication (Read, 2000).

However, in order to understand the process of vocabulary learning observing only lexical issues does not suffice. Other factors influencing lexical development must be taken into consideration, which include learners' engagement and action, which assumes learners' ability to employ learning strategies and tactics to regulate their learning (Tseng \& Schmitt, 2008: 358).

The present study is an attempt to explore the role that self-regulated vocabulary learning may play in lexical competence of learners of English as a foreign language (EFL). 
The paper is organized as follows: first, the concepts of language learning strategies and selfregulated learning are defined and explained, then the lexical dimensions pertinent to the study are outlined. The study is described in section 2 . The conclusions, implications and future research paths close the paper.

\subsection{Language learning strategies vs. self-regulated learning}

When learning a foreign language, learners adopt numerous language learning strategies, i.e. "actions chosen by learners (either deliberately or automatically) for the purpose of learning or regulating the learning of language" (Griffiths, 2015, p. 426). Language learning strategies (LLSs) have raised considerable interest among experts in the field of second language acquisition (SLA), which has led to establishing a strong research community committed to studying them. Forty years of research of LLSs has generated significant insights into this aspect of learners' individual differences, depicting what learners do in order to tackle language learning problems (cf. Macaro, 2006; Cohen \& Macaro 2007; Griffiths \& Oxford, 2014). Nevertheless, it has also attracted criticism due to a range of pending issues such as the lack of a unanimous definition of the construct of strategy, problematic classifications and inadequate research instruments (cf. Dörnyei, 2005; Rose, 2012a; 2012b).

The ongoing argument about the exact nature of LLSs and the methods of studying them led to a rather revolutionary proposal put forward by Dörnyei (2005) who suggested the focus of research should be on studying a trait, i.e. students' aptitude for strategic learning, rather than on specific learning behaviours (i.e. LLSs) which are simply the result of aptitude-driven efforts. He referred to this aptitude as self-regulated learning (SRL), drawing on the legacy of a well-established research field in education and psychology that has only recently extended its reach to the second language acquisition (SLA) research (Collett, 2014). A widely accepted definition of SRL describes it as "an active, constructive process whereby learners set goals for their learning and then attempt to monitor, regulate, and control their cognition, motivation, and behaviour, guided and constrained by their goals and the contextual features in the environment" (Pintrich, 2000, p. 453). SLA theorists have discussed self-regulation as a broader construct which, in addition to LLSs, also includes autonomy, metacognition, motivation, and self-management (Chamot, 2014). Gao (2007) believes that the study of self-regulated learning should not lead to replacing but rather complementing LLS research, where self-regulation measures the initial force and the strategy research measures the outcomes. Likewise, Oxford $(2011,2017)$ has incorporated the concept of self-regulation into the existing paradigms of strategies, emphasizing that on the one hand, strategies are vital elements of most theories of self-regulation and on the other hand, self-regulation is the major purpose of LLS use (Oxford, 2017).

As a result of new research possibilities opening up by introducing the construct of self-regulation LLSs research has taken a number of directions identified by Rose et al. 
(2018): (i) study of self-regulation has replaced LLS research; (ii) self-regulation is acknowledged within existing conceptualizations of LLS (e.g. existing instruments have been adapted to provide psychometrically-sound measures of LLSs); and (iii) LLS research is reconceptualized (theory from both self-regulation and LLS research is considered). Not surprisingly, these findings indicate that LLS research has not been terminated by the introduction of the concept of self-regulation because LLSs "are the raw material of learner agency and a key to understanding achievement, or the lack thereof" (Ranalli, 2012, p. 373) and, as specific learning behaviours, will continue to draw attention in spite of difficulties of defining the right approach to measuring them.

This study sets out to research the concept of self-regulation as students' aptitude to be strategic about their vocabulary learning, as envisaged by Dörnyei (2005), and embodied in the Tseng et al.'s (2006) seminal study focusing on the development of a new instrument for measuring self-regulation in vocabulary learning. The instrument, called Self-regulatory Capacity for Vocabulary Learning (SRCvoc) (Tseng et al., 2006), embodies the proposed idea that conceptualizing and assessing strategic learning should be based on the learners' innate self-regulatory capacity, i.e. a trait, rather than the specific strategic behaviours they engage in while learning. It is a questionnaire limited to measuring self-regulatory capacity in the domain of vocabulary learning, following the authors' conviction that each domain should be treated separately because it is not possible to design an instrument to embrace all language aspects. The structure and content of the questionnaire are based on taxonomies of action control strategies developed by Dörnyei (2001), thus the 20 items measure five different facets of self-regulatory capacity for vocabulary learning, each measured by 4 items of the questionnaire. Commitment control refers to the learners' ability to preserve or increase their commitment to achieving the original goal (e.g. Item 10: When learning vocabulary, I persist until I reach the goals that I make for myself.). Metacognitive control involves monitoring and controlling of concentration and reducing procrastination (e.g. Item 5: When learning vocabulary, I have special techniques to keep my concentration focused.). Satiation control indicates the ability to eliminate boredom and to add extra appeal to the task (e.g. Item 1: Once the novelty of learning vocabulary is gone, I easily become impatient with it.). Emotion control pertains to eliminating turbulent emotions or moods, and generating emotions more conducive to learning (e.g. Item 6: I feel satisfied with the methods I use to reduce the stress of vocabulary learning.). Environmental control concerns managing environmental influences by making them supportive in attaining the set goals (e.g. Item 20: When learning vocabulary, I look for a good learning environment.).

Rose et al.'s (2018) findings show that this approach to LLS research has thus far been limited to the validation or adaptation studies of this work indicating that the potential of this approach has not yet been fully exploited. This study will attempt to partly fill this gap in research by providing some answers pertaining to the relationship of self-regulated vocabulary learning and lexical competence. 


\subsection{Aspects of lexical knowledge}

One of the analytical frameworks for researching the development of lexical competence, put forward by Bulté et al. (2008), is shown in Picture 1. Lexical proficiency is defined as a cognitive construct consisting of the declarative and the procedural component. Procedural lexical competence, comprising learners' control over this knowledge, accounts for the manner of online lexical access, retrieval and (de)coding. The declarative component, entailing the components of size, width and depth of lexical knowledge, accounts for much of the complexity of lexical knowledge. Because these theoretical constructs cannot be observed or measured, conclusions must be drawn on the basis of the manifestations of the cognitive constructs in learners' comprehension and production. Thus, the declarative component is operationalised via the lower-order constructs of lexical diversity, sophistication, complexity and productivity.

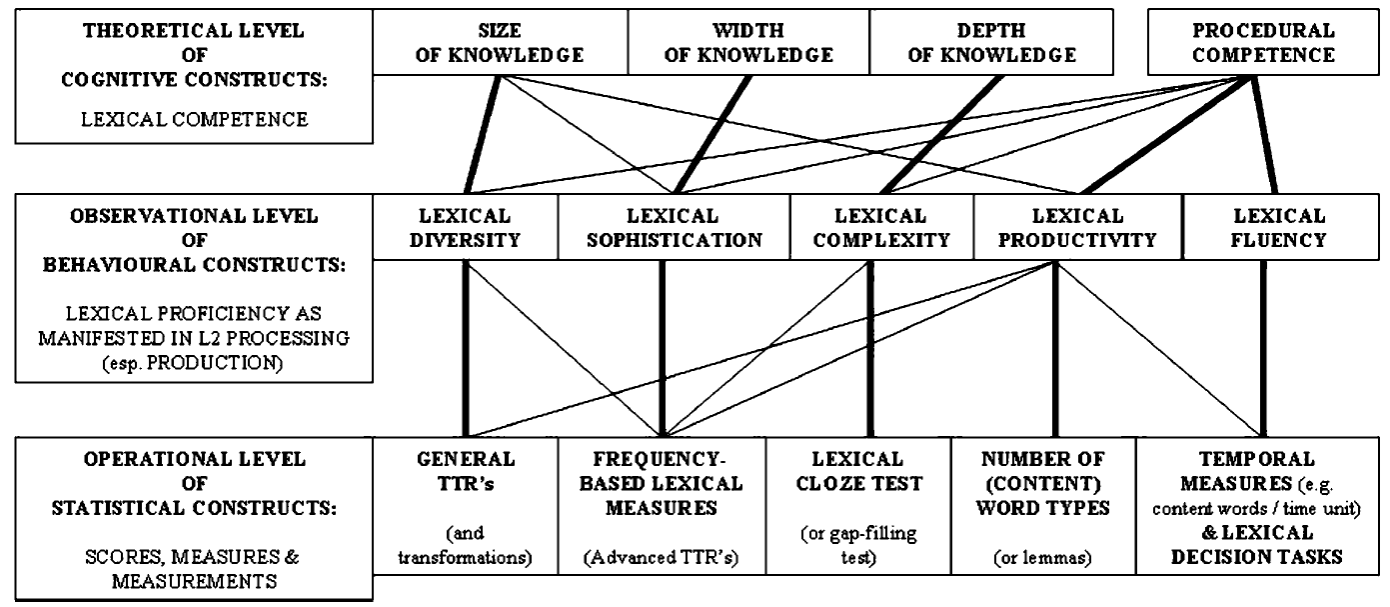

Figure 1. Analytical framework for researching the development of lexical competence

(from Bulté et al. 2008)

The behavioural constructs adopted for this study were lexical density, lexical diversity and lexical sophistication. This triad of interrelated aspects of lexical competence have been used elsewhere in research to depict the multidimensional feature of learners' language production referred to as lexical richness (Wolfe-Quintero et al., 1998; Read, 2000). Read's (2000) conceptualization of lexical richness also involves the fourth element - lexical errors - that are, however, not in the focus of the current research. The construct of lexical richness has been proved to relate directly to the learners' ability to engage in effective spoken and written communication (Lu, 2012) which makes it a significant construct in SLA research. The terminology allocated to the constructs adopted for this research is not yet fully stable. Thus, what is indicated as lexical productivity in Bulté et al.'s (2008) framework is also known as lexical density in other research (e.g. Lu, 2012), and it stands for the ratio 
of the number of lexical words to the total number of words in a text. Lexical diversity, also known as lexical variation (Read, 2000; Lu, 2012) refers to the range of learners' vocabulary as displayed in their language use. Finally, lexical sophistication or lexical rareness is "the proportion of relatively unusual or advanced words in the learner's text" (Read, 2000, p. 203).

The advance of research on lexical richness has been accompanied by the development of a range of improved measures, i.e. statistical constructs that operationalise the various components of lexical richness. Research pertaining to these statistical indices has indicated the most reliable ones (Lu, 2012; McCarthy and Jarvis, 2007, 2010), which were selected for inclusion in this study (Picture 2). As can be seen, there is a wide range of measures of lexical diversity. The original measures of lexical diversity (e.g. number of different words - NDW; type token ratio - TTR) proved to be sensitive to the length of language sample which prompted pursuit of more reliable measures, often based on different sampling techniques. Taking into account lexical sophistication as one of the measures puts focus on the frequency with which particular words are generally used in a language. A larger number of low-frequency words used in a text would indicate a higher level of vocabulary knowledge. Such approach was adopted by Laufer and Nation (1995) in designing their Lexical Frequency Profile (LFP) which calculates the proportion of word types in a text at different levels of frequency (in the first 1,000 most frequent words, the second 1,000 most frequent words, the university word list and those not included in any of these three).

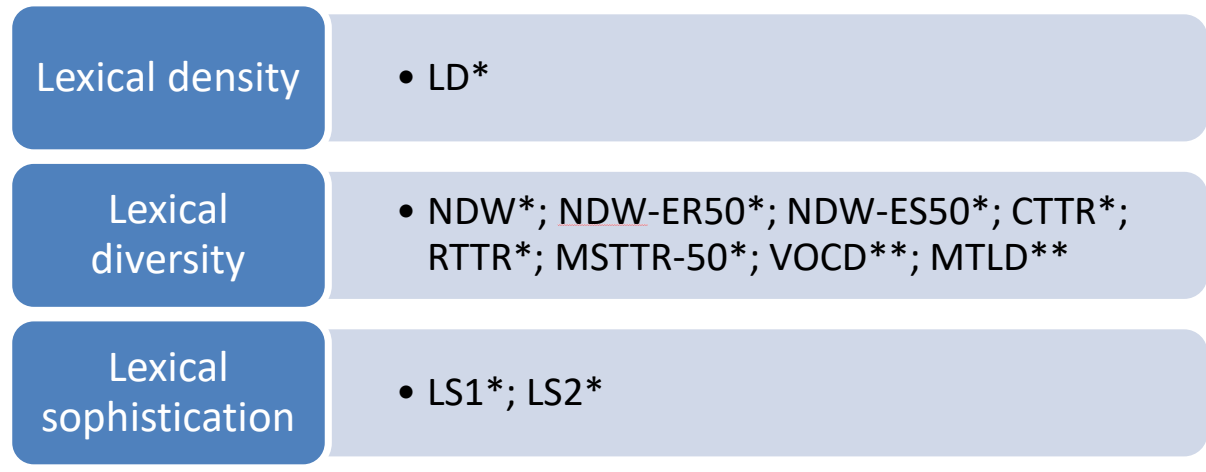

Figure 2. * - according to Lu, 2012; ${ }^{* *}$ - according to McCarthy and Jarvis, 2007, 2010

\section{The study}

\subsection{Aim}

The main aim of this study is to explore the role of self-regulated vocabulary learning in students' productive lexical competence. Accordingly, there are two research questions that the study will try to answer: 
RQ 1: Is there a correlation between students' self-regulatory capacity for vocabulary learning and lexical diversity, density and sophistication of their writing?

RQ 2: How much of the variance in lexical competence scores can be explained by the five dimensions of self-regulation in vocabulary learning (i.e. commitment control, metacognitive control, satiation control, emotion control and environmental control): Which of these facets is a better predictor of each of the indicators of lexical competence, i.e. a) lexical diversity, b) lexical density, and c) lexical sophistication?

\subsection{Instruments}

In order to provide answers to the two research questions the study applied three instruments:

1) Self-regulating capacity in Vocabulary Learning Scale (SRCvoc) (Tseng et al., 2006); 2) measures of lexical diversity, density and sophistication as operationalisations of the construct of lexical competence; and 3) students' written texts.

SRCvoc is a questionnaire measuring self-regulated capacity for vocabulary learning. The items in the questionnaire are worded as general declarations or conditional relations and there are no specific strategic behaviours mentioned. The response to the questionnaire items is given on a 6-item Likert scale ranging from "strongly agree" to "strongly disagree". In statistical data analysis attention should be paid to the two negatively worded items that require re-coding ( 1 and 12$)$. The questionnaire items $(\alpha=0.845)$ form five subscales: commitment control $(\alpha=0.592)$, metacognitive control $(\alpha=0.663)$, satiation control $(\alpha=$ 0.557), emotion control ( $\alpha=0.654)$, and environment control $(\alpha=0.507)$. The reliability coefficient Cronbach alpha as measured for each scale is lower in this study than in the original questionnaire (commitment: $\alpha=0.81$; metacognitive: 0.71 ; satiation: 0.80 ; emotion: 0.82; environment: 0.74 ). The replications conducted in Japan and Iran also showed lower Cronbach alpha values than the original ones (commitment: 0.63 in Japan and 0.65 in Iran; metacognitive: 0.74 in Japan and 0.66 in Iran; satiation: 0.71 in Japan and 0.42 in Iran; emotion: 0.66 in Japan and 0.69 in Iran; environment: 0.67 in Japan and 0.65 in Iran) (for details see Mizumoto and Takeutchi, 2012; Doaee et al., 2017). The Turkish validation of SRCvoc reported on the overall Cronbach alpha $(\alpha=0.89)$ but did not provide reliability coefficients of each particular subscale (Yeşilbursa \& Bilican, 2013). However, the authors did report on problems with several items and concluded although SRCvoc proved to be a reliable and valid instrument "it may be be sensitive to cultural differences, and hence further studies need to be conducted in different cultural contexts with participants of different ages to shed more light on the concept." (Yeşilbursa \& Bilican, 2013: 885).

As for lexical knowledge, this study has opted for measuring the productive knowledge given the fact that the study participants have a relatively high level of English 
proficiency and often claim they have a higher receptive than productive command of the language. The analysis of students' lexical competence was conducted within the analytical framework for researching the development of lexical competence put forward by Bulte et al. (2008) (cf. Picture 1). As has been stated, the behavioural constructs included in this study were lexical density, lexical diversity and lexical sophistication. Lexical density (LD) was measured by a single index which stands for the ratio of the number of lexical words to the total number of words in a text. Based on Lu (2012) the following indices of lexical diversity were included in the study: NDW (number of different words), NDW-ER50 (expected random 50; calculated as the mean number of different words of 10 random 50-word samples), NDW-ES50 (expected sequence 50; calculated as the mean number of different words of 10 random 50-word sequences), CTTR (corrected type token ratio), RTTR (root type token ratio), MSTTR-50 (mean type token ratio of all 50-word segments). Two more measures were added following McCarthy and Jarvis $(2007,2010)$ who are strong advocates of VOCD, calculated through random sampling or sequential sampling, and MTLD (measure of textual lexical diversity), "calculated as the mean length of sequential word strings in a text that maintain a given TTR value" (McCarthy \& Jarvis, 2010, p. 384). The authors consider these to be more robust approaches to lexical diversity assessment and yet they advise researchers to use a combination of indices rather than any single index. Two indices of lexical sophistication were used in this study: LS1 stands for the ratio of sophisticated lexical words to the total number of lexical words while LS2 refers to the ratio of the sophisticated word types to the total number of word types in a text ( $\mathrm{Lu}, 2012)$. The statistical indices were calculated using the online tools described below (see section 2.4.).

In order to obtain a sample of students' real language for which the described lexical measures can be taken we opted for eliciting free production in form of written compositions, the form whose level of complexity allows us to distinguish between more proficient and less proficient writers (Laufer, 1998).

\subsection{Participants and data collection}

The participants of this study were 84 students of the Tourism study programmes at the Faculty of Economics, Business and Tourism, University of Split. The study involved both students of undergraduate $(\mathrm{N}=48)$ and professional study $(\mathrm{N}=36)$ programmes who took English for Specific Purposes (English in Tourism 1 and 2) as obligatory courses in the first two semesters. The current syllabi for these courses include a series of written assignments related to the studied topics. One of the assignments, related to the topic of accommodation, required students to write a text describing property (real or imagined) to be advertised on Airbnb. The text structure was provided in form of the subtitles following the Airbnb's guidelines for property description: The space, Guest access, Interaction with guests, The

neighbourhood, Getting around. Besides offering scaffolding to the inexperienced writers 
this structure was also meant to make the texts more comparable. The length requirement was between 300 and 500 words. The length of the 84 analysed texts ranged from 139 to 616 words. For the needs of this study the task was performed under test conditions to ensure the texts were actual students' free production. The writing took place on the computers in the IT lab where students were logged in the exam mode. Such setup connects all students' computers to the central one (that of a researcher) making it possible for students to access the task and to submit the task in a common folder which facilitates the process of task administration (allocation and submission). More importantly, this setup does not allow importing any external documents or information and it does not allow access to any external sources of information (e.g. the Internet).

SRCvoc was administered on a different occasion. In order to ensure students' anonymity but be able to match the two submissions (the text and the questionnaire) we asked participants to code their contributions following these guidelines: $1^{\text {st }}$ letter in their mother's name, $1^{\text {st }}$ letter in their father's name, F or M (for female or male), date of birth (two digits).

The following section explains how we approached data analysis and what tools were used for that purpose.

\subsection{Data analysis}

The statistical analysis of the data was performed using SPSS 23.0 (2015). In particular, the correlations between the five facets of self-regulatory capacity of vocabulary learning and the selected indices of lexical density, diversity and sophistication were calculated. Also, we ran a series of regression analyses with all lexical measures as dependent variables.

Two lexical density measures (VOCD and MTLD) were calculated using the Text Inspector (2018) while the rest of statistical indices described in 2.2.2. were generated using the Lexical Complexity Analyzer (Ai \& Lu, 2010). Before submitting the texts to the automatic analysis performed by these two online tools they had to be pre-processed: numbers were removed; proper nouns were deleted (e.g. names of cities, streets, restaurants, etc.); spelling errors were corrected. Such methodological decisions were made because keeping the removed items would have affected the results and/or their interpretation (see for example Granger \& Wynne, 1999). The latter two interventions were to ensure that the automatic analysis programmes did not recognise the proper nouns and spelling mistakes as rare words, thus mistakenly increasing the values of the calculated indices. For example, the word lists applied in measuring sophistication do not contain names of the cities and would classify these words as rare and the text itself would have an inflated lexical sophistication score. The same is true of spelling mistakes which would produce the words not included in any of the frequency word lists. For the same reason the texts would also seem to be characterised by higher lexical density and diversity. 


\section{Results}

In order to answer the research question 1 correlation analysis was run between the five facets of self-regulatory capacity for vocabulary learning and all the statistical indices of lexical density, diversity and sophistication selected for this study. The results are given in Table 1.

Table 1. Correlations between five facets of the self-regulatory capacity for vocabulary learning and measures of lexical density, diversity and sophistication $(\mathrm{N}=84)$

\begin{tabular}{lccccc}
\hline & commitment & metacognitive & satiation & emotion & environmental \\
\hline lexical density & .063 & .063 & .150 & .213 & .014 \\
NDW & .129 & .186 & $.253^{*}$ & .202 & .057 \\
NDWERZ & -.070 & -.096 & -.077 & -.105 & -.015 \\
NDWESZ & -.200 & -.043 & -.043 & -.066 & .030 \\
MSTTR & -.143 & -.053 & .036 & -.048 & -.047 \\
CTTR & -.031 & .046 & .069 & .068 & -.057 \\
RTTR & .075 & .088 & .167 & .149 & -.007 \\
VOCD & -.074 & -.040 & -.034 & -.029 & .020 \\
MTLD & -.098 & -.044 & -.007 & -.030 & .005 \\
LS1 & .039 & .057 & .128 & .084 & .100 \\
LS2 & .057 & .050 & .154 & .144 & -.033 \\
\hline
\end{tabular}

${ }^{*} \mathrm{p}<0.05$

As indicated by the results only one correlation showed low statistical significance at the 0.05 level $(\mathrm{r}=.253)$. This is the correlation between satiation control and one of the measures of lexical diversity (NDW - number of different words). This result could potentially indicate that the students who are better able to eliminate boredom and to add extra appeal to the task when learning vocabulary also have a wider range of vocabulary as displayed in their language use. This is, however, not supported by any other of the 
remaining 7 measures of lexical diversity. The rest of the correlations were not significant. This leads us to conclude that there is no correlation between the five facets of selfregulatory capacity of vocabulary learning and the students' lexical competence operationalised by the three measures of lexical richness (lexical density, lexical diversity, and lexical sophistication).

Next, in order to answer the research question 2 we ran a number of regression analyses with all lexical measures as dependent variables and SRCvoc facets as predictors. In total, eleven regression models were run. As shown in Table 2 the variance in the dependent variances explained by the models is minute (between 0.7 and $7 \%$ ). None of the models were significant and none of the predictors reached statistical significance which means that SRCvoc facets are not predictors of lexical competence as measured by these indices.

Table 2. Results of the regression analysis with the range of lexical measures as dependent variables and five facets of the self-regulatory capacity for vocabulary learning as independent variables

\begin{tabular}{lllcl}
\hline Dependent variable & $\mathbf{R}$ & $\mathbf{R}^{\mathbf{2}}$ & adjusted $\mathbf{R}^{\mathbf{2}}$ & \multicolumn{1}{l}{ Sig } \\
\hline lexical density & .272 & .074 & .015 & .296 \\
NDW & .153 & .023 & -.039 & .865 \\
NDWERZ & .214 & .046 & -.016 & .591 \\
NDWESZ & .085 & .007 & -.056 & .989 \\
MSTTR & .110 & .012 & -.051 & .965 \\
CTTR & .268 & .072 & .012 & .315 \\
RTTR & .112 & .013 & -.051 & .963 \\
VOCD & .229 & .053 & -.008 & .509 \\
MTLD & .161 & .026 & -.036 & .836 \\
LS1 & .199 & .040 & -.022 & .668 \\
LS2 & .185 & .034 & -.028 & .736 \\
\hline
\end{tabular}




\section{Discussion and conclusion}

In this paper we addressed the issue of the relationship of lexical competence and selfregulatory capacity of vocabulary learning. It was assumed that a greater ability to selfregulate ones' vocabulary learning would lead to greater vocabulary gains, i.e. higher lexical proficiency. Furthermore, it was assumed that this relationship would best be captured by measuring lexical richness of free productive use of language on the one hand, and applying a psychometrically valid measure of self-regulation on the other hand.

The results showed that there was no correlation between students' self-regulatory capacity (measured by SRCvoc) and lexical density, diversity and sophistication of their writing (measured by LD, NDW, NDW-ER50, NDW-ES50, CTTR, RTTR, MSTTR-50, VOCD, MTLD, LS1, LS2). In other words, the results suggest that students with a higher lexical competence (operationalised as the three measures of lexical richness) are not those who perceive themselves as being more highly self-regulated in vocabulary learning and vice versa, those students who achieved higher scores on the self-regulation instrument did not produce lexically richer texts. Accordingly, the regression analyses ran with the selected lexical measures as dependent variables showed that self-regulation as measured by SRCvoc is not a predictor of lexical competence. These findings imply that factors other than selfregulation as conceptualised in the SRCvoc might have stronger influence on lexical proficiency. Learner-dependent factors that have a stronger predictive power may well be vocabulary learning strategies, i.e. actual behaviours/actions students adopt when learning vocabulary. According to Gu (2018) this could also be more useful since SRCvoc - being a measure of learner's capacity for self-management and control - lacks the ability of indicating the actual ways in which vocabulary should best be learned.

\section{Limitations of the study}

The findings of the present study could be attributed to the possible limitations of the study. These limitations also indicate the way forward for future research in this field.

Not finding any correlation between the aptitude for being strategic about one's vocabulary learning (the "trait approach") and the lexical competence calls for reintroducing the study of strategies students employ in vocabulary learning, as has been indicated above. This would necessitate introducing a strategy inventory questionnaire in the study design.

Next, as already mentioned accuracy is an important element of lexical competence but was not the focus of this study. Adding lexical error analysis would generate one more variable whose relationship with the five self-regulation facets could be explored thus potentially providing a deeper insight into the role of self-regulation in lexical competence. 
Also, instead of individual lexical measures, a more holistic assessment score of lexical competence as the dependent variable might yield different results.

Furthermore, the characteristics of the present sample may have played a role in the study's outcome. The respondents in this study were non-language majors who had been exposed to many years of both instructed and naturalistic English language learning. This may have resulted in the decline of self-regulation of vocabulary learning or the lack of awareness of strategies used to this end, as a result of a relatively high language proficiency. It appears possible that the results may be different in studies involving participants at lower levels of language learning or at different age levels, or those of different professions and cultural backgrounds. Having a larger sample may also prove beneficial.

Finally, one cannot disregard the possibility that the underlying self-regulation model stemming from the volitional theory of self-regulation does not optimally account for language learners' approaches to vocabulary learning. Therefore, an attempt can be made to look at self-regulation in vocabulary learning using a different theoretical model (for examples of self-regulation theories and models see e.g. Ranalli, 2012; Panadero, 2017).

\section{References:}

Ai, H. \& Lu, X. (2010). A web-based system for automatic measurement of lexical complexity. Paper presented at the 27th Annual Symposium of the Computer-Assisted Language Consortium (CALICO-10). Amherst, MA. June 8-12.

Bulté, B., Housen, A., Pierrard M. \& Van Daele, S. (2008). Investigating lexical proficiency development over time - the case of Dutch speaking learners of French in Brussels. Journal of French Language Studies, 18, 277-298.

Chamot, A. U. (2014). Developing self-regulated learning in the language classroom. In W. M. Chan, K. N. Chin, \& T. Suthiwan (Eds.), Proceedings of CLaSIC 2014 (pp. 78-88). Paper presented at the sixth CLS international conference CLaSIC 2014. Singapore: Knowledge, Skills and Competencies in Foreign Language Education.

Cohen, A. \& Macaro, E. (Eds.). (2007). Language learner strategies: Thirty years of research and practice. Oxford: Oxford University Press.

Collett, P. (2014). Researching self-regulated learning and foreign language learning. Studies in Self-Access Learning Journal, 5(4), 430-442.

Daller, H., Milton, J. \& Treffers-Daller, J. (2007). Editors' introduction: Conventions, terminology and an overview of the book. In H. Daller, J. Milton \& J. Treffers-Daller (Eds.), Modelling and Assessing Vocabulary Knowledge (pp. 1-32). Cambridge: Cambridge University Press.

Doaee, M.T., Sarkeshikian, S.A.H, \& Tabatabaee, S.A.M. (2017). Investigating the Reliability and Factor Structure of the Self-Regulating Capacity in Vocabulary Learning (SRCvoc) 
in Iranian EFL Context. The Journal of English Language Pedagogy and Practice. 10(20), 169-186.

Dörnyei, Z. (2001). Motivational Strategies in the Language Classroom. Cambridge: Cambridge University Press.

Dörnyei, Z. (2005). Psychology of the language learner: Individual differences in second language acquisition. London and New York: Lawrence Erlbaum Associates.

Gao, X. (2007). Has language learning strategy research come to an end? A response to Tseng et al. (2006). Applied Linguistics, 28(4), 615-620.

Granger, S. \& Wynne, M. (1999). Optimising measures of lexical variation in EFL learner corpora. In J. Kirk (Ed.), Corpora Galore (pp. 249-257). Amsterdam and Atlanta: Rodopi.

Griffiths, C. \& Oxford, R. (2014). The twenty-first century landscape of language learning strategies: Introduction to this special issue. System 43: 1-10.

Griffiths, C. (2015). What have we learnt from 'good language learners'? ELT Journal, 69(4), 425-433.

$\mathrm{Gu}, \mathrm{Y}$. (2018). Validation of an online questionnaire of vocabulary learning strategies for ESL learners. Studies in Second Language Learning and Teaching, 8(2), 325-350.

IBM Corp. Released 2015. IBM SPSS Statistics for Windows, Version 23.0. Armonk, NY: IBM Corp.

Laufer, B. (1998). The development of passive and active vocabulary in a second language: same or different? Applied Linguistics, 19(2), 255-271.

Laufer, B., \& Nation, P. (1995). Vocabulary Size and Use: Lexical Richness in L2 Written Production. Applied Linguistics, 16(3), 307-322.

Lu, X. (2012). The Relationship of Lexical Richness to the Quality of ESL Learners' Oral Narratives. The Modern Language Journal, 96(2), 190-208.

Macaro, E. (2006). Strategies for language learning and for language use: revising the theoretical framework. Modern Language Journal 90 (3): 320-337.

McCarthy, P.M. \& Jarvis, S. (2007). vocd: A theoretical and empirical evaluation. Language Testing 24(4), 459-488.

McCarthy, P.M. \& Jarvis, S. (2010). MTLD, vocd-D, and HD-D: A validation study of sophisticated approaches to lexical diversity assessment. Behavior Research Methods $42(2), 381-392$.

Milton, J. (2009). Measuring Second Language Vocabulary Acquisition. Bristol: Multilingual Matters.

Mizumoto, A. \& Takeutchi, O. (2012). Adaptation and Validation of Self-regulating Capacity in Vocabulary Learning Scale. Applied Linguistics 33(1),83-91.

Nation, I. S. P. (2001). Learning vocabulary in another language. Cambridge: Cambridge University Press. 
Oxford, R. (2011). Teaching and researching language learning strategies. Harlow: Pearson ESL.

Oxford, R. (2017). Teaching and researching language learning strategies: Self-regulation in context. New York: Routledge.

Panadero, E. (2017). A Review of Self-regulated Learning: Six Models and Four Directions for Research. Frontiers in Psychology, 8:422.

Pintrich, P. R. (2000). The role of goal orientation in self-regulated learning. In Boekaerts, M., Pintrich, P. R. and Zeidner, M. (Eds.), Handbook of Self-Regulation (pp. 451-502). San Diego: Academic Press.

Ranalli, J. (2012). Alternative models of self-regulation and implications for L2 strategy research. Studies in Self-Access Learning Journal, 3(4), 357-376.

Read, J. (2000). Assessing Vocabulary. Cambridge: Cambridge University Press.

Rose, H. (2012a). Reconceptualizing Strategic Learning in the Face of Self-Regulation: Throwing Language Learning Strategies out with the Bathwater. Applied Linguistics 33 (1), 92-98.

Rose, H. (2012b). Language learning strategy research: Where do we go from here? Studies in Self-Access Learning Journal 3 (2), 137-148.

Rose, H., Briggs, J. G., Boggs, J. A., Sergio, L., \& Ivanova-Slavianskaia, N. (2018). A systematic review of language learner strategy research in the face of self-regulation. System, 72, 151-163.

Text Inspector (2018). Online lexis analysis tool at textinspector.com [Accessed 04/03/2018]

Tseng, W. \& Schmitt, N. (2008). Toward a model of motivated vocabulary learning: A structural equation modeling approach. Language Learning 58 (2), 357-400.

Tseng, W., Dörnyei, Z., \& Schmitt, N. (2006). A New Approach to Assessing Strategic Learning: The Case of Self-Regulation in Vocabulary Acquisition. Applied Linguistics, 27(1), 78102.

Wolfe-Quintero, K., S. Inagaki \& H.-Y. Kim. (1998). Second Language Development in Writing: Measures of Fluency, Accuracy, and Complexity. Honolulu, HI: University of Hawai'i, Second Language Teaching and Curriculum Center.

Yeşilbursa, A., \& Bilican, R. (2013). Validation of self-regulatory capacity for vocabulary Learning Scale in Turkish. Procedia: Social-Behavioral Sciences, 70, 882-886. 